\title{
Angiolymphoid Hyperplasia with Eosinophilia- A Case Report
}

\author{
Pankaj Kumar Doloi ${ }^{1}$, Swagata Khanna ${ }^{2}$ \\ ${ }^{1}$ ENT, Head \& Neck Clinic, Swagat ESRI, Guwahati, Assam, India \\ ${ }^{2}$ Department of ENT, Gauhati Medical College \& Hospital, Guwahati, Assam, India \\ Email: doloi.pankaj@gmail.com
}

Received June 1, 2012; revised July 5, 2012; accepted August 2, 2012

\begin{abstract}
Angiolymphoid hyperplasia with eosinophilia is a rare, benign vascular tumor affecting principally the head and neck region of young adult females. Microscopic analysis reveals hyperplastic blood vessels lined by a hypertrophic endothelium. An inflammatory infiltrate rich in eosinophils is also present. Etiology of the lesion is unknown. Various treatment modalities have been described. We present a case successfully treated by excision and local steroid infiltration.
\end{abstract}

Keywords: Angiolymphoid Hyperplasia with Eosinophilia (ALHE); Epithelioid Haemangioma; Histiocytoid Haemangioma; Kimura's Disease

\section{Introduction}

Angiolymphoid hyperplasia with eosinophilia (ALHE, epithetlioid haemangioma, inflammatory angiomatous nodule, atypical granuloma, pseudopyogenic granuloma, and histiocytoid hemangioma) is an uncommon, benign, reactive vaso-proliferative disease, presenting with painless, vascular nodules in the dermal and subcutaneous tissues of the head and neck, particularly around the ear [1]. ALHE has also been reported in the scalp, lip tongue, orbits and the conjunctiva [1-4]. Although frequency is unknown, cases have been reported worldwide. This condition is uncommon but not rare. It may be more common in Japan than in other countries. ALHE can persist for years, but serious complications (e.g., malignant transformation) do not occur and have never been reported. ALHE is seen most commonly in Asian, followed by Caucasian. Although less commonly blacks too can develop ALHE. It is rare in elderly patients and in the non-Asian paediatric population. ALHE is somewhat more common in females; however, a male predominance has been noted in selected Asian studies [5]. It presents most commonly in patients aged 20 - 50 years, with a mean onset of 30 - 33 years [5].

ALHE is characterized clinically by single to multiple red brown dome shaped papules or subcutaneous nodules [1-4]. About 1/5 of patients have blood eosinophilia and Lymphadenopathy [2]. Although it is usually superficial in nature, some authors have reported muscular and bony involvement [1-4,6].

\section{Case Report}

An eighteen-year-old Hindu female attended our outpatient department with complaint of a gradually increasing painless swelling in the left frontal region extending to the eyebrow of one year's duration (Figure 1). The skin over other parts of her body did not show any abnormality. She had similar type of scalp swelling on the same side six months back for which she got operated in a general hospital. The histopathology report came as reactive hyperplasia. After a period of three months a similar swelling reappeared at the same site and it was again operated in the same hospital without any definitive diagnosis. She did not give a positive history for fever, skin rashes, asthma and respiratory infection, or any history of louse infestation of the scalp since childhood. On clinical examination there was no evidence of Lymphadenopathy or hepatosplenomegaly. The left frontal region showed a tumour mass, $3 \mathrm{~cm} \times 2.5 \mathrm{~cm} \times 1 \mathrm{~cm}$ in size, firm in consistency and subcutaneous in situation. The swelling was non-tender with restricted mobility. Clinical impression of epidermoid cyst of the scalp was made.

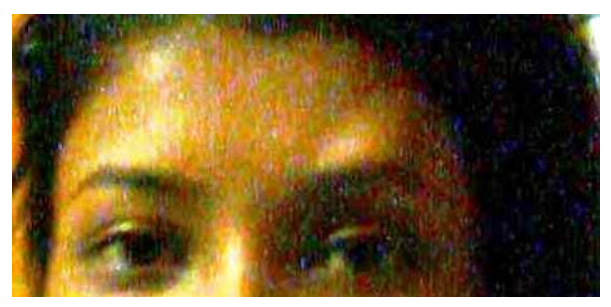

Figure 1. Picture of the patient. 
Routine blood and urine investigations were within normal limit. Chest screening showed no abnormality. Complete surgical excision of the mass was performed under general anaesthesia by maintaining the aesthetic aspect of the surgery (Figure 2). The excised specimen was examined histopathologically. It was followed by local infiltration of injection Triamcelone Acetate after one month and three months over the lesion. After seven months of follow up there is no recurrence.

The size of the tumour was $2.5 \mathrm{~cm} \times 2 \mathrm{~cm} \times 1 \mathrm{~cm}$. The external surface was smooth. On cut surface, the tumour showed fine whitish capsule surrounding pale pink areas. Microscopy revealed lobular proliferation of thick and thin walled blood vessels lined by plump endothelial cells and separated by an inflamed fibrocollagenous stroma. The inflammation comprises of lymphocytes, neutrophils and many eosinophils. Few lymphoid follicles are also seen (Figures 3 and 4).

\section{Discussion}

Angiolymphoid hyperplasia with eosinophilia (ALHE) is a rare condition affecting muscular arteries, typically of the head and neck [1]. It was first described in 1969 by Wells and Whimster [4]. They reported nine patients between the ages of 19 and 43, five women and four men, with single to multiple lesions in the head and neck region with blood eosinophillia in all patients and regional lymphadenopathy in four of nine patients. Previously it had been described as pseudo- or atypical pyogenic granuloma, subcutaneous angioblastic lymphoid hyperplasia with eosinophilia, and papular angioplasia $[1,6]$. Initially it was thought to be related to Kimura's disease but recent histological studies indicate that Kimura disease differs from angiolymphoid hyperplasia with eosinophilia in several clinical and histopathologic characteristics, including male predominance, striking lymphadenopathy, higher incidence of peripheral blood eosinophilia, and lack of the distinctive endothelial cell as a marker [2,6-8].

Histologically the lesions are characterized by a reactive proliferation of small blood vessels, often surrounding a muscular artery, with peripheral inflammatory infiltrates consisting of mononuclear cells and eosinophils. The reactive blood vessels are often epithelioid, leading to the terms "histiocytoid" or, more recently "epithelioid" haemangioma [6]. Immunohistochemical stains usually show a major population of T-Lymphocytes [5] with occasional B cells forming lymphoid follicles [6]. Since the description of the initial large series [6], there have been numerous reports of this condition, with lesions occurring in a variety of organs, including disseminated disease [1,9-15].

The pathogenesis of ALHE remains unclear. Some authors consider ALHE as a neoplasm developing from

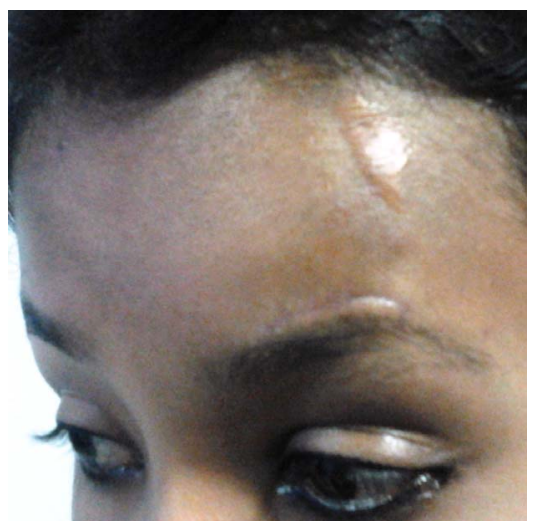

Figure 2. Forehead scar of previous surgery and present scar over the left eyebrow.

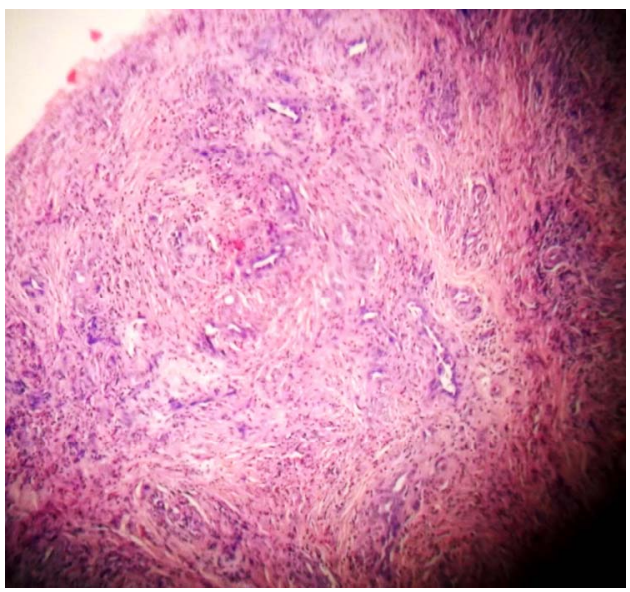

Figure 3. Low power microscopic view.

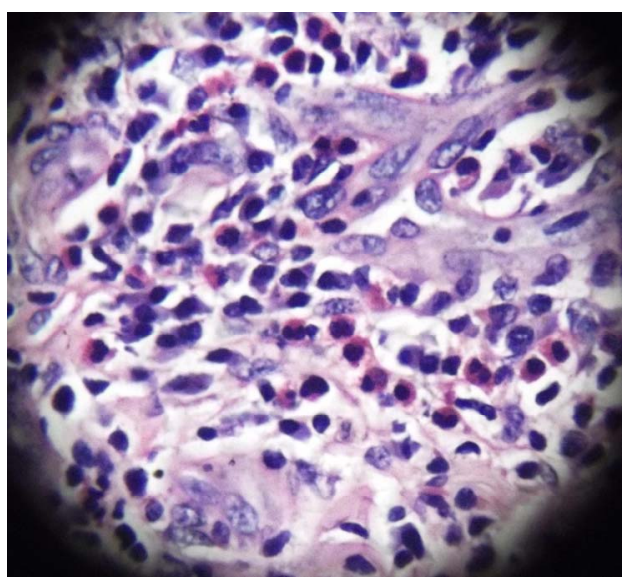

Figure 4. High power microscopic view showing aggregate of lymphoid tissue and numerous proliferated blood vessels with plump endothelial cells and eosinophils in the background (H \& E stain).

endothelial cells; others suggest that it is secondary to an inflammatory vascular reaction secondary to complex immunologic mechanisms. Many other hypotheses have been reported implicating environmental factors such as 
insect bite, trauma, and infections. Some authors consider that arterio-venous shunt is the main etiopathogenetic mechanism observed in 42 percent of the cases $[7,16]$. The predominance of $\mathrm{T}$ lymphocytes and a rearrangement of TCR receptor in some cases made some authors suppose that ALHE is a low-grade neoplastic disease secondary to various stimuli $[7,16]$.

Serum hypereosinophilia is inconstant (21\%) and is not required to make the diagnosis. Given that some patients with ALHE have also been found to have renal disease, urinalysis could be considered. Radiologic examinations such as MRI or angiography may be required to determine the extension of the lesions $[7,16]$. Positive diagnosis is based upon histological findings. Other differential diagnosis based on clinical and/or histopathologic findings, includes insect bites, angiomatous neoplasias such as capillary hemangioma, granuloma pyogenicum with satellite lesions, angiosarcoma of the face and scalp, and Kaposi sarcoma. Furthermore, epidermal cysts, lymphadenosis cutis benigna, and granuloma faciale might mimic angiolymphoid hyperplasia with eosinophilia.

In the absence of treatment, lesions may either increase progressively or decrease spontaneously [12]. Surgical treatment remains the treatment of choice. Recurrences, essentially after incomplete excision, are observed in 30 percent of the cases [7]. No metastatic cases have been reported $[7,16]$. Considering the possible spontaneous involution of the lesions, a simple follow up is recommended 3 to 6 months before surgical excision or other extensive therapeutic modalities are attempted. Many therapeutic procedures have been described in the literature including electro-dessication, cryotherapy, micrographic surgery, systemic corticoid treatment, intra-lesional injection of corticoids or sclerosing products, phototherapy, or alpha-2a interferon. Despite the multiplicity of therapeutic modalities, their real efficacy has not been well studied and frequent recurrences have been noted. Surgical excision may be efficient in limited lesions but recurrences remain frequent because surgical margins remain difficult to determine.

Interleukin-5 based treatment represents an interesting approach. This cytokine interferes with the production and activation of eosinophils, which are supposed to play a key role in the pathogenesis of ALHE. Imiquimod, which inhibits the production of interleukin 5, and mepolizumab, which inhibits the reaction of IL5 with its receptor, were reported to be effective [17].

\section{Conclusion}

Angiolymphoid hyperplasia with eosinophilia is a rare condition with a challenging diagnosis and treatment. In spite of the benignity of this disease, it causes a therapeutic dilemma because of the cosmetic defects and frequent resistance to treatment.

\section{REFERENCES}

[1] M. V. Botet and J. L. Sanchez, “Angiolymphoid Hyperplasia with Eosinophilia: Report of a Case and a Review of the Literature," Journal of Dermatologic Surgery and Oncology, Vol. 4, No. 12, 1978, pp. 931-936.

[2] W. Kempf, A. C. Haeffner, K. Zepter, C. A. Sander, M. J. Flaig, B. Mueller, R. G. Panizzon, T. Hardmeier, V. Adams and G. Burg, "Angiolymphoid Hyperplasia with Eosinophilia: Evidence for a T-Cell Lymphoproliferative Origin,” Human Pathology, Vol. 33, No. 10, 2002, pp. 10231029. doi:10.1053/hupa.2002.128247

[3] A. V. Peña, E. de D. Rodríguez, J. M. G. Ortega, A. H. Castrillo and G. L. Rasines, "Considerations about Angiolymphoid Hyperplasia with Eosinophilia (ALHE) with Regard to a Case Localized in the Penis,” Actas Urológicas Españolas, Vol. 29, No. 1, 2005, pp. 113-117. doi:10.4321/S0210-48062005000100020

[4] G. C. Wells and I. W. Whimster, "Subcutaneous Angiolymphoid Hyperplasia with Eosinophilia,” British Journal of Dermatology, Vol. 81, No. 1, 1969, pp. 1-15. doi:10.1111/j.1365-2133.1969.tb15914.X

[5] C. A. Moran and S. Suster, “Angiolymphoid Hyperplasia with Eosinophilia (Epithelioid Hemangioma) of the Lung: A Clinicopathologic and Immunohistochemical Study of Two Cases," American Journal of Clinical Pathology, Vol. 123, No. 5, 2005, pp. 762-765. doi:10.1309/UN1AQ2WJU9HDD72F

[6] T. G. Olsen and E. B. Helwig, “Angiolymphoid Hyperplasia with Eosinophilia: A Clinicopathologic Study of 116 Patients," Journal of American Academy of Dermatology, Vol. 12, No. 5, 1985, pp. 781-796. doi:10.1016/S0190-9622(85)70098-9

[7] P. L. Ramchandani, T. Sabesan and K. Hussein, “Angiolymphoid Hyperplasia with Eosinophilia Masquerading as Kimura Disease,” British Journal of Oral Maxillofacial Surgery, Vol. 43, No. 3, 2005, pp. 249-252.

doi:10.1016/j.bjoms.2004.11.023

[8] W.-S. Chong, A. Thomas and C.-L. Goh, "Kimura’s Disease and Angiolymphoid Hyperplasia with Eosinophilia: Two Disease Entities in the Same Patient. Case Report and Review of the Literature," International Journal of Dermatology, Vol. 45, No. 2, 2006, pp. 139-145. doi:10.1111/j.1365-4632.2004.02361.x

[9] A. Acocella, C. Catelani and P. Nardi, “Angiolymphoid Hyperplasia with Eosinophilia: A Case Report of Orbital Involvement,” Journal of Oral and Maxillofacial Surgery, Vol. 63, No. 1, 2005, pp. 140-144.

doi:10.1016/j.joms.2004.04.029

[10] M. Azizzadeh, M. R. Namazi, L. Dastghaib and F. SariAslani, “Angiolymphoid Hyperplasia with Eosinophilia and Nephrotic Syndrome," International Journal of Dermatology, Vol. 44, No. 3, 2005, pp. 242-244. doi:10.1111/j.1365-4632.2004.02030.x

[11] R. K. Hejmadi, D. G. van Pittius, M. Stephens, R. Chasty and M. Braithwaite, "Angiolymphoid Hyperplasia with Eosinophilia (Epithelioid Haemangioma) Occurring within Multiple Deep Lymph Nodes and Presenting with Weight Loss and Raised CA-125 Levels," Virchows Archiv, Vol. 448, No. 3, 2005, pp. 366-368. 
[12] A. Satpathy, C. Moss, F. Raafat and R. Slator, "Spontaneous Regression of a Rare Tumour in a Child: Angiolymphoid Hyperplasia with Eosinophilia of the Hand: Case Report and Review of the Literature," British Journal of Plastic Surgery, Vol. 58, No. 6, 2005, pp. 865-868. doi:10.1016/j.bjps.2004.11.014

[13] H. Suzuki, A. Hatamochi, M. Horie, T. Suzuki and S. Yamazaki, "A Case of Angiolymphoid Hyperplasia with Eosinophilia (ALHE) of the Upper Lip,” Journal of Dermatology, Vol. 32, No. 12, 2005, pp. 991-995.

[14] N. Zarrin-Khameh, J. E. Spoden and R. M. Tran, “Angiolymphoid Hyperplasia with Eosinophilia Associated with Pregnancy: A Case Report and Review of the Literature," Archives of Pathology \& Laboratory Medicine,
Vol. 129, No. 9, 2005, pp. 1168-1171.

[15] G. Y. Zhang, J. Jiang, T. Lin and Q. Q. Wang, “Disseminated Angiolymphoid Hyperplasia with Eosinophilia: A Case Report,” Cutis, Vol. 72, No. 4, 2003, pp. 323-326.

[16] T. Demitsu, H. Nagato and T. Inoue, “Angiolymphoid Hyperplasia with Eosinophilia: Its Character and Therapy,” Skin Surgery, Vol. 9, No. 1, 2000, pp. 8-16.

[17] M. Braun-Falco, S. Fischer, S.-G. Plötz and J. Ring, “Angiolymphoid Hyperplasia with Eosinophilia Treated with Anti-Interleukin-5 Antibody (Mepolizumab),” British Journal of Dermatology, Vol. 151, No. 5, 2004, pp. 11031104. doi:10.1111/j.1365-2133.2004.06239.x 